Article

\title{
Geometric Properties of Certain Analytic Functions Associated with the Dziok-Srivastava Operator
}

\section{Cai-Mei Yan ${ }^{1}$ and Jin-Lin Liu ${ }^{2, *}$}

1 Information Engineering College, Yangzhou University, Yangzhou 225002, China; cmyan@yzu.edu.cn

2 Department of Mathematics, Yangzhou University, Yangzhou 225002, China

* Correspondence: jlliu@yzu.edu.cn

Received: 20 January 2019; Accepted: 15 February 2019; Published: 19 February 2019

Abstract: The objective of the present paper is to derive certain geometric properties of analytic functions associated with the Dziok-Srivastava operator.

Keywords: analytic function; subordination; Dziok-Srivastava operator

2010 Mathematics Subject Classification: 30C45

\section{Introduction}

Throughout this paper, we assume that:

$$
n, p \in \mathbb{N},-1 \leq B<A \leq 1, \alpha>0 \text { and } \beta<1
$$

Let $A_{n}(p)$ denote the class of functions of the form:

$$
f(z)=z^{p}+\sum_{k=n+p}^{\infty} a_{k} z^{k}
$$

which are analytic in the open unit disk $U=\{z:|z|<1\}$. If $f(z)=z^{p}+\sum_{k=n+p}^{\infty} a_{k} z^{k} \in A_{n}(p)$ and $g(z)=z^{p}+\sum_{k=n+p}^{\infty} b_{k} z^{k} \in A_{n}(p)$, then the Hadamard product (or convolution) of $f$ and $g$ is defined by:

$$
(f * g)(z)=z^{p}+\sum_{k=n+p}^{\infty} a_{k} b_{k} z^{k}
$$

For:

$$
\alpha_{j} \in \mathbb{C}(j=1,2, \cdots, l) \text { and } \beta_{j} \in \mathbb{C} \backslash\{0,-1,-2, \cdots\}(j=1,2, \cdots, m)
$$

the generalized hypergeometric function ${ }_{l} F_{m}\left(\alpha_{1}, \cdots, \alpha_{l} ; \beta_{1}, \cdots, \beta_{m} ; z\right)$ is defined by:

$$
\begin{gathered}
{ }_{l} F_{m}\left(\alpha_{1}, \cdots, \alpha_{l} ; \beta_{1}, \cdots, \beta_{m} ; z\right)=\sum_{k=0}^{\infty} \frac{\left(\alpha_{1}\right)_{k} \cdots\left(\alpha_{l}\right)_{k}}{\left(\beta_{1}\right)_{k} \cdots\left(\beta_{m}\right)_{k}} \frac{z^{k}}{k !} \\
\left(l \leq m+1 ; l, m \in \mathbb{N}_{0}=\mathbb{N} \cup\{0\} ; z \in U\right)
\end{gathered}
$$

where $(x)_{k}$ is the Pochhammer symbol given by $(x)_{k}=x(x+1) \cdots(x+k-1)$ for $k \in \mathbb{N}$ and $(x)_{0}=1$. Corresponding to the function $z^{p}{ }_{l} F_{m}\left(\alpha_{1}, \cdots, \alpha_{l} ; \beta_{1}, \cdots, \beta_{m} ; z\right)$, the well-known Dziok-Srivastava operator [1] $H\left(\alpha_{1}, \cdots, \alpha_{l} ; \beta_{1}, \cdots, \beta_{m}\right): A_{n}(p) \rightarrow A_{n}(p)$ is defined by:

$$
H\left(\alpha_{1}, \cdots, \alpha_{l} ; \beta_{1}, \cdots, \beta_{m}\right) f(z)=\left(z^{p_{l}} F_{m}\left(\alpha_{1}, \cdots, \alpha_{l} ; \beta_{1}, \cdots, \beta_{m} ; z\right) * f(z)\right.
$$




$$
\left(l \leq m+1 ; l, m \in \mathbb{N}_{0} ; z \in U\right)
$$

If $f \in A_{n}(p)$ is given by (2), then we have:

$$
H\left(\alpha_{1}, \cdots, \alpha_{l} ; \beta_{1}, \cdots, \beta_{m}\right) f(z)=z^{p}+\sum_{k=n+p}^{\infty} \frac{\left(\alpha_{1}\right)_{k} \cdots\left(\alpha_{l}\right)_{k}}{\left(\beta_{1}\right)_{k} \cdots\left(\beta_{m}\right)_{k}} \frac{a_{k}}{k !} z^{k}
$$

For convenience, we write:

$$
H_{m}^{l}\left(\alpha_{1}\right)=H\left(\alpha_{1}, \cdots, \alpha_{l} ; \beta_{1}, \cdots, \beta_{m}\right) \quad\left(l \leq m+1 ; l, m \in \mathbb{N}_{0}\right)
$$

It is noteworthy to mention that the Dziok-Srivastava operator is a generalization of certain linear operators considered in earlier investigations.

Next, we consider the function $h(A, B ; z)=(1+A z) /(1+B z)$ for $z \in U$. It is known that the function $h(A, B ; z)$ is the conformal map of $U$ onto a disk, symmetrical with respect to the real axis, which is centered at the point $(1-A B) /\left(1-B^{2}\right)(B \neq \pm 1)$ and with its radius equal to $(A-B) /$ $\left(1-B^{2}\right)(B \neq \pm 1)$. Furthermore, the boundary circle of this disk intersects the real axis at the points $(1-A) /(1-B)$ and $(1+A) /(1+B)$ with $B \neq \pm 1$.

Let $P[A, B]$ denote the class of functions of the form $p(z)=1+p_{1} z+\cdots$, which are analytic in $U$ and satisfy the subordination $p(z) \prec h(A, B ; z)$. It is clear that $p \in P[A, B]$ if and only if:

$$
\left|p(z)-\frac{1-A B}{1-B^{2}}\right|<\frac{A-B}{1-B^{2}} \quad(-1<B<A \leq 1 ; z \in U)
$$

and:

$$
\operatorname{Re} p(z)>\frac{1-A}{2} \quad(B=-1 ; z \in U)
$$

For two functions $f$ and $g$ analytic in $U, f$ is said to be subordinate to $g$, written by $f(z) \prec$ $g(z) \quad(z \in U)$, if there exists a Schwarz function $w$ in $U$ such that:

$$
|w(z)| \leq|z| \quad \text { and } \quad f(z)=g(w(z)) \quad(z \in U)
$$

Furthermore, if the function $g$ is univalent in $U$, then:

$$
f(z) \prec g(z) \quad(z \in U) \Longleftrightarrow f(0)=g(0) \quad \text { and } \quad f(U) \subset g(U)
$$

Many properties of analytic functions have been investigated by several authors(see [1-11]). In this paper, we derive certain geometric properties of analytic functions associated with the well-known Dziok-Srivastava operator.

\section{Main Results}

Theorem 1. Let $f$ belong to the class $A_{n}(p)$. Furthermore, let:

$$
\frac{H_{m}^{l}\left(\alpha_{1}\right) f(z)}{z^{p}} \in P[A, B]
$$

Then:

$$
\begin{aligned}
& \operatorname{Re}\left\{\frac{H_{m}^{l}\left(\alpha_{1}\right) f(z)}{z^{p}}+\alpha z\left(\frac{H_{m}^{l}\left(\alpha_{1}\right) f(z)}{z^{p}}\right)^{\prime}\right\} \\
& \leq \begin{cases}\frac{1+(A+B+n \alpha(A-B)) r^{n}+A B r^{2 n}}{\left(1+B r^{n}\right)^{2}} & \text { if } M_{n}(A, B, \alpha, r) \leq 0, \\
\frac{L_{n}^{2}-4 \alpha^{2} K_{A} K_{B}}{4 \alpha(A-B) r^{n-1}\left(1-r^{2}\right) K_{B}} & \text { if } M_{n}(A, B, \alpha, r) \geq 0,\end{cases}
\end{aligned}
$$


where:

$$
\left\{\begin{array}{l}
K_{A}=1-A^{2} r^{2 n}+n A r^{n-1}\left(1-r^{2}\right), \\
K_{B}=1-B^{2} r^{2 n}+n B r^{n-1}\left(1-r^{2}\right), \\
L_{n}=2 \alpha\left(1-A B r^{2 n}\right)+n \alpha(A+B) r^{n-1}\left(1-r^{2}\right)+(A-B) r^{n-1}\left(1-r^{2}\right), \\
M_{n}(A, B, \alpha, r)=2 \alpha K_{B}\left(1+A r^{n}\right)-L_{n}\left(1+B r^{n}\right) .
\end{array}\right.
$$

The result is sharp.

Proof. For $z=0$, the equality in (4) holds true. Thus, we assume that $0<|z|=r<1$. From (3), we can write:

$$
\frac{H_{m}^{l}\left(\alpha_{1}\right) f(z)}{z^{p}}=\frac{1+A z^{n} \varphi(z)}{1+B z^{n} \varphi(z)} \quad(z \in U),
$$

where $\varphi(z)$ is analytic and $|\varphi(z)| \leq 1$ in $U$. From (7), we have:

$$
\begin{aligned}
\frac{H_{m}^{l}\left(\alpha_{1}\right) f(z)}{z^{p}}+\alpha z\left(\frac{H_{m}^{l}\left(\alpha_{1}\right) f(z)}{z^{p}}\right)^{\prime} \\
=\frac{H_{m}^{l}\left(\alpha_{1}\right) f(z)}{z^{p}}+\frac{\alpha(A-B) z^{n}\left(n \varphi(z)+z \varphi^{\prime}(z)\right)}{\left(1+B z^{n} \varphi(z)\right)^{2}} \\
\quad=\frac{H_{m}^{l}\left(\alpha_{1}\right) f(z)}{z^{p}}+\frac{n \alpha}{A-B}\left(A-B H_{m}^{l}\left(\alpha_{1}\right) f(z) / z^{p}\right)\left(H_{m}^{l}\left(\alpha_{1}\right) f(z) / z^{p}-1\right) \\
\quad+\frac{\alpha(A-B) z^{n+1} \varphi^{\prime}(z)}{\left(1+B z^{n} \varphi(z)\right)^{2}}
\end{aligned}
$$

By using the Carathéodory inequality:

$$
\left|\varphi^{\prime}(z)\right| \leq \frac{1-|\varphi(z)|^{2}}{1-r^{2}}
$$

we get:

$$
\begin{aligned}
\operatorname{Re}\left\{\frac{z^{n+1} \varphi^{\prime}(z)}{\left(1+B z^{n} \varphi(z)\right)^{2}}\right\} & \leq \frac{r^{n+1}\left(1-|\varphi(z)|^{2}\right)}{\left(1-r^{2}\right)\left|1+B z^{n} \varphi(z)\right|^{2}} \\
& =\frac{r^{2 n}\left|A-B H_{m}^{l}\left(\alpha_{1}\right) f(z) / z^{p}\right|^{2}-\left|H_{m}^{l}\left(\alpha_{1}\right) f(z) / z^{p}-1\right|^{2}}{(A-B)^{2} r^{n-1}\left(1-r^{2}\right)}
\end{aligned}
$$

Set $\frac{H_{m}^{l}\left(\alpha_{1}\right) f(z)}{z^{p}}=u+i v \quad(u, v \in \mathbb{R})$. Then, (8) and (9) give:

$$
\begin{aligned}
& \operatorname{Re}\left\{\frac{H_{m}^{l}\left(\alpha_{1}\right) f(z)}{z^{p}}+\alpha z\left(\frac{H_{m}^{l}\left(\alpha_{1}\right) f(z)}{z^{p}}\right)^{\prime}\right\} \\
& \leq\left(1+n \alpha \frac{A+B}{A-B}\right) u-\frac{n \alpha A}{A-B}-\frac{n \alpha B}{A-B}\left(u^{2}-v^{2}\right) \\
&+\alpha \frac{r^{2 n}\left((A-B u)^{2}+(B v)^{2}\right)-\left((u-1)^{2}+v^{2}\right)}{(A-B) r^{n-1}\left(1-r^{2}\right)} \\
&=\left(1+n \alpha \frac{A+B}{A-B}\right) u-\frac{n \alpha}{A-B}\left(A+B u^{2}\right) \\
&+\alpha \frac{r^{2 n}(A-B u)^{2}-(u-1)^{2}}{(A-B) r^{n-1}\left(1-r^{2}\right)}+\frac{\alpha}{A-B}\left(n B-\frac{1-B^{2} r^{2 n}}{r^{n-1}\left(1-r^{2}\right)}\right) v^{2}
\end{aligned}
$$


Note that:

$$
\begin{aligned}
\frac{1-B^{2} r^{2 n}}{r^{n-1}\left(1-r^{2}\right)} & \geq \frac{1-r^{2 n}}{r^{n-1}\left(1-r^{2}\right)}=\frac{1}{r^{n-1}}\left(1+r^{2}+r^{4}+\cdots+r^{2(n-2)}+r^{2(n-1)}\right) \\
& =\frac{1}{2 r^{n-1}}\left[\left(1+r^{2(n-1)}\right)+\left(r^{2}+r^{2(n-2)}\right)+\cdots+\left(r^{2(n-1)}+1\right)\right] \\
& \geq n \geq n B
\end{aligned}
$$

Using (10) and (11), we obtain:

$$
\begin{aligned}
\operatorname{Re}\left\{\frac{H_{m}^{l}\left(\alpha_{1}\right) f(z)}{z^{p}}+\alpha z\left(\frac{H_{m}^{l}\left(\alpha_{1}\right) f(z)}{z^{p}}\right)^{\prime}\right\} & \leq\left(1+n \alpha \frac{A+B}{A-B}\right) u-\frac{n \alpha}{A-B}\left(A+B u^{2}\right) \\
& +\alpha \frac{r^{2 n}(A-B u)^{2}-(u-1)^{2}}{(A-B) r^{n-1}\left(1-r^{2}\right)}=\psi_{n}(u)
\end{aligned}
$$

It is known that for $|\xi| \leq \sigma(\sigma<1)$,

$$
\left|\frac{1+A \xi}{1+B \xi}-\frac{1-A B \sigma^{2}}{1-B^{2} \sigma^{2}}\right| \leq \frac{(A-B) \sigma}{1-B^{2} \sigma^{2}}
$$

and:

$$
\frac{1-A \sigma}{1-B \sigma} \leq \operatorname{Re}\left\{\frac{1+A \xi}{1+B \xi}\right\} \leq \frac{1+A \sigma}{1+B \sigma}
$$

Furthermore, (7) and (14) show that:

$$
\frac{1-A r^{n}}{1-B r^{n}} \leq \operatorname{Re}\left\{\frac{H_{m}^{l}\left(\alpha_{1}\right) f(z)}{z^{p}}\right\} \leq \frac{1+A r^{n}}{1+B r^{n}} .
$$

Now, we calculate the maximum value of $\psi_{n}(u)$ on the segment $\left[\frac{1-A r^{n}}{1-B r^{n}}, \frac{1+A r^{n}}{1+B r^{n}}\right]$. Obviously,

$$
\begin{gathered}
\psi_{n}^{\prime}(u)=1+n \alpha \frac{A+B}{A-B}-\frac{2 n \alpha B}{A-B} u+2 \alpha \frac{\left(1-A B r^{2 n}\right)-\left(1-B^{2} r^{2 n}\right) u}{(A-B) r^{n-1}\left(1-r^{2}\right)} \\
\psi_{n}^{\prime \prime}(u)=-\frac{2 \alpha}{A-B}\left(n B+\frac{1-B^{2} r^{2 n}}{r^{n-1}\left(1-r^{2}\right)}\right)<0 \quad \text { (see (11)) }
\end{gathered}
$$

and $\psi_{n}^{\prime}(u)=0$ if and only if:

$$
\begin{aligned}
u=u_{n}= & \frac{2 \alpha\left(1-A B r^{2 n}\right)+n \alpha(A+B) r^{n-1}\left(1-r^{2}\right)+(A-B) r^{n-1}\left(1-r^{2}\right)}{2 \alpha\left[1-B^{2} r^{2 n}+n B r^{n-1}\left(1-r^{2}\right)\right]} \\
& =\frac{L_{n}}{2 \alpha K_{B}} \quad(\text { see (6) })
\end{aligned}
$$

Since:

$$
\begin{aligned}
& 2 \alpha K_{B}\left(1-A r^{n}\right)-L_{n}\left(1-B r^{n}\right) \\
& \quad=2 \alpha\left[\left(1-A r^{n}\right)\left(1-B^{2} r^{2 n}\right)-\left(1-B r^{n}\right)\left(1-A B r^{2 n}\right)\right] \\
& \quad-n \alpha r^{n-1}\left(1-r^{2}\right)\left[(A+B)\left(1-B r^{n}\right)-2 B\left(1-A r^{n}\right)\right]-(A-B) r^{n-1}\left(1-r^{2}\right)\left(1-B r^{n}\right) \\
& \quad=-2 \alpha(A-B) r^{n}\left(1-B r^{n}\right)-n \alpha(A-B) r^{n-1}\left(1-r^{2}\right)\left(1+B r^{n}\right) \\
& \quad-(A-B) r^{n-1}\left(1-r^{2}\right)\left(1-B r^{n}\right)<0
\end{aligned}
$$

we see that:

$$
u_{n}>\frac{1-A r^{n}}{1-B r^{n}}
$$


However, $u_{n}$ is not always less than $\frac{1+A r^{n}}{1+B r^{n}}$. The following two cases arise.

Case (I). $u_{n} \geq \frac{1+A r^{n}}{1+B r^{n}}$, that is $M_{n}(A, B, \alpha, r) \leq 0$. In view of $\psi_{n}^{\prime}\left(u_{n}\right)=0$ and (15), the function $\psi_{n}(u)$ is increasing on the segment $\left[\frac{1-A r^{n}}{1-B r^{n}}, \frac{1+A r^{n}}{1+B r^{n}}\right]$. Thus, we deduce from (12) that, if $M_{n}(A, B, \alpha, r) \leq 0$, then:

$$
\begin{aligned}
\operatorname{Re}\left\{\frac{H_{m}^{l}\left(\alpha_{1}\right) f(z)}{z^{p}}+\alpha z\left(\frac{H_{m}^{l}\left(\alpha_{1}\right) f(z)}{z^{p}}\right)^{\prime}\right\} & \leq \psi_{n}\left(\frac{1+A r^{n}}{1+B r^{n}}\right) \\
& =\left(1+n \alpha \frac{A+B}{A-B}\right)\left(\frac{1+A r^{n}}{1+B r^{n}}\right) \\
& -\frac{n \alpha}{A-B}\left(A+B\left(\frac{1+A r^{n}}{1+B r^{n}}\right)^{2}\right) \\
& =\frac{1+A r^{n}}{1+B r^{n}}-\frac{n \alpha}{A-B}\left(1-\frac{1+A r^{n}}{1+B r^{n}}\right)\left(A-B \frac{1+A r^{n}}{1+B r^{n}}\right) \\
& =\frac{1+(A+B+n \alpha(A-B)) r^{n}+A B r^{2 n}}{\left(1+B r^{n}\right)^{2}}
\end{aligned}
$$

This gives (4).

Next, we consider the function $f$ defined by:

$$
\frac{H_{m}^{l}\left(\alpha_{1}\right) f(z)}{z^{p}}=\frac{1+A z^{n}}{1+B z^{n}}
$$

which satisfies the condition (3). It is easy to check that:

$$
\frac{H_{m}^{l}\left(\alpha_{1}\right) f(r)}{r^{p}}+\alpha r\left(\frac{H_{m}^{l}\left(\alpha_{1}\right) f(r)}{r^{p}}\right)^{\prime}=\frac{1+(A+B+n \alpha(A-B)) r^{n}+A B r^{2 n}}{\left(1+B r^{n}\right)^{2}}
$$

which implies that the inequality (4) is sharp.

Case (II). $u_{n} \leq \frac{1+A r^{n}}{1+B r^{n}}$, that is $M_{n}(A, B, \alpha, r) \geq 0$. In this case, we easily have:

$$
\operatorname{Re}\left\{\frac{H_{m}^{l}\left(\alpha_{1}\right) f(z)}{z^{p}}+\alpha z\left(\frac{H_{m}^{l}\left(\alpha_{1}\right) f(z)}{z^{p}}\right)^{\prime}\right\} \leq \psi_{n}\left(u_{n}\right)
$$

In view of (6), $\psi_{n}(u)$ in (12) can be written as:

$$
\psi_{n}(u)=\frac{-\alpha K_{B} u^{2}+L_{n} u-\alpha K_{A}}{(A-B) r^{n-1}\left(1-r^{2}\right)}
$$

Therefore, if $M_{n}(A, B, \alpha, r) \geq 0$, then it follows from (16), (18), and (19) that:

$$
\begin{aligned}
\operatorname{Re}\left\{\frac{H_{m}^{l}\left(\alpha_{1}\right) f(z)}{z^{p}}+\alpha z\left(\frac{H_{m}^{l}\left(\alpha_{1}\right) f(z)}{z^{p}}\right)^{\prime}\right\} & \leq \frac{-\alpha K_{B} u_{n}^{2}+L_{n} u_{n}-\alpha K_{A}}{(A-B) r^{n-1}\left(1-r^{2}\right)} \\
& =\frac{L_{n}^{2}-4 \alpha^{2} K_{A} K_{B}}{4 \alpha(A-B) r^{n-1}\left(1-r^{2}\right) K_{B}}
\end{aligned}
$$

To show the sharpness, we take:

$$
\frac{H_{m}^{l}\left(\alpha_{1}\right) f(z)}{z^{p}}=\frac{1+A z^{n} \varphi(z)}{1+B z^{n} \varphi(z)} \quad \text { and } \quad \varphi(z)=\frac{z-c_{n}}{1-c_{n} z}
$$


where $c_{n} \in \mathbb{R}$ is determined by:

$$
\frac{H_{m}^{l}\left(\alpha_{1}\right) f(r)}{r^{p}}=\frac{1+A r^{n} \varphi(r)}{1+B r^{n} \varphi(r)}=u_{n} \in\left(\frac{1-A r^{n}}{1-B r^{n}}, \frac{1+A r^{n}}{1+B r^{n}}\right]
$$

Clearly, $-1<\varphi(r) \leq 1,-1 \leq c_{n}<1,|\varphi(z)| \leq 1(z \in U)$, and so, $f$ satisfies the condition (3). Since:

$$
\varphi^{\prime}(r)=\frac{1-c_{n}^{2}}{\left(1-c_{n} r\right)^{2}}=\frac{1-|\varphi(r)|^{2}}{1-r^{2}}
$$

from the above argument, we find that:

$$
\frac{H_{m}^{l}\left(\alpha_{1}\right) f(r)}{r^{p}}+\alpha r\left(\frac{H_{m}^{l}\left(\alpha_{1}\right) f(r)}{z^{p}}\right)^{\prime}=\psi_{n}\left(u_{n}\right)
$$

The proof of the theorem is now completed.

Corollary 1. Let $f \in A_{1}(p)$, and satisfy $\operatorname{Re}\left\{H_{m}^{l}\left(\alpha_{1}\right) f(z) / z^{p}\right\}>\beta(\beta<1 ; z \in U)$. Then, for $|z|=r<1$,

$$
\operatorname{Re}\left\{\frac{H_{m}^{l}\left(\alpha_{1}\right) f(z)}{z^{p}}+\alpha z\left(\frac{H_{m}^{l}\left(\alpha_{1}\right) f(z)}{z^{p}}\right)^{\prime}\right\} \leq \beta+(1-\beta) \frac{1+2 \alpha r-r^{2}}{(1-r)^{2}}
$$

The result is sharp.

Proof. By considering $\frac{H_{m}^{l}\left(\alpha_{1}\right) f(z) / z^{p}-\beta}{1-\beta}$ instead of $H_{m}^{l}\left(\alpha_{1}\right) f(z) / z^{p}$, we only need to prove the corollary for $\beta=0$. Putting $n=A=1$ and $B=-1$ in (6), we get:

$$
K_{1}=2\left(1-r^{2}\right), \quad K_{-1}=0, \quad L_{1}=2 \alpha\left(1+r^{2}\right)+2\left(1-r^{2}\right)
$$

and:

$$
M_{1}(1,-1, \alpha, r)=-2(1-r)\left[1+\alpha-(1-\alpha) r^{2}\right] \leq 0
$$

Consequently, an application of (4) in Theorem 2.1 yields:

$$
\operatorname{Re}\left\{\frac{H_{m}^{l}\left(\alpha_{1}\right) f(z)}{z^{p}}+\alpha z\left(\frac{H_{m}^{l}\left(\alpha_{1}\right) f(z)}{z^{p}}\right)^{\prime}\right\} \leq \frac{1+2 \alpha r-r^{2}}{(1-r)^{2}}
$$

The sharpness follows immediately from that of Theorem 1.

Theorem 2. Let $\alpha_{j}(j=1,2, \cdots, l)$ and $\beta_{s}(s=1,2, \cdots, m)$ be positive real numbers. Furthermore, let $f(z)=z^{p}+\sum_{k=n+p}^{\infty} a_{k} z^{k} \in A_{n}(p)$, and satisfy:

$$
\frac{H_{m}^{l}\left(\alpha_{1}\right) f(z)}{z^{p}}+\alpha z\left(\frac{H_{m}^{l}\left(\alpha_{1}\right) f(z)}{z^{p}}\right)^{\prime} \in P[A, B]
$$

Then:

$$
\left|a_{k}\right| \leq \frac{k !(A-B)\left(\beta_{1}\right)_{k} \cdots\left(\beta_{m}\right)_{k}}{(1+\alpha(k-p))\left(\alpha_{1}\right)_{k} \cdots\left(\alpha_{l}\right)_{k}} \quad(k \geq n+p)
$$

The result is sharp for each $k \geq n+p$.

Proof. It is well known that if:

$$
g(z)=\sum_{k=1}^{\infty} b_{k} z^{k} \prec \varphi(z) \quad(z \in U)
$$


where $g(z)$ is analytic in $U$ and $\varphi(z)=z+\cdots$ is convex univalent in $U$, then $\left|b_{k}\right| \leq 1(k=1,2,3, \cdots)$.

From (20), we have:

$$
\begin{aligned}
\frac{1}{A-B}\left(\frac{H_{m}^{l}\left(\alpha_{1}\right) f(z)}{z^{p}}+\alpha z\left(\frac{H_{m}^{l}\left(\alpha_{1}\right) f(z)}{z^{p}}\right)^{\prime}-1\right) \\
=\frac{1}{A-B} \sum_{k=n+p}^{\infty} \frac{(1+\alpha(k-p))\left(\alpha_{1}\right)_{k} \cdots\left(\alpha_{l}\right)_{k} \cdot a_{k}}{k !\left(\beta_{1}\right)_{k} \cdots\left(\beta_{m}\right)_{k}} z^{k-p} \\
\quad \prec \frac{z}{1+B z} \quad(z \in U)
\end{aligned}
$$

In view of the function $\frac{z}{1+B z}$ being convex univalent in $U$, it follows from (22) that:

$$
\frac{(1+\alpha(k-p))\left(\alpha_{1}\right)_{k} \cdots\left(\alpha_{l}\right)_{k}}{k !(A-B)\left(\beta_{1}\right)_{k} \cdots\left(\beta_{m}\right)_{k}}\left|a_{k}\right| \leq 1 \quad(k \geq n+p)
$$

which gives (21).

Next, we consider the function $f_{k-p}(z)$ defined by:

$$
f_{k-p}(z)=z^{p}+(A-B) \sum_{q=1}^{\infty} \frac{(-B)^{q-1}\left(\beta_{1}\right)_{q k} \cdots\left(\beta_{m}\right)_{q k}(q k) !}{(1+\alpha q(k-p))\left(\alpha_{1}\right)_{q k} \cdots\left(\alpha_{l}\right)_{q k}} z^{q(k-p)+p} \quad(z \in U ; k \geq n+p)
$$

Since:

$$
\frac{H_{m}^{l}\left(\alpha_{1}\right) f_{k-p}(z)}{z^{p}}+\alpha z\left(\frac{H_{m}^{l}\left(\alpha_{1}\right) f_{k-p}(z)}{z^{p}}\right)^{\prime}=\frac{1+A z^{k-p}}{1+B z^{k-p}} \prec \frac{1+A z}{1+B z} \quad(z \in U)
$$

and:

$$
f_{k-p}(z)=z^{p}+\frac{k !(A-B)\left(\beta_{1}\right)_{k} \cdots\left(\beta_{m}\right)_{k}}{(1+\alpha(k-p))\left(\alpha_{1}\right)_{k} \cdots\left(\alpha_{l}\right)_{k}} z^{k}+\cdots
$$

for each $k \geq n+p$, the proof of Theorem 2 is completed.

Author Contributions: All authors contributed equally.

Funding: This work is supported by the National Natural Science Foundation of China (Grant No. 11571299).

Conflicts of Interest: The authors declare no conflict of interest.

\section{References}

1. Dziok, J.; Srivastava, H.M. Classes of analytic functions associated with the generalized hypergeometric function. Appl. Math. Comput. 1999, 103,1-13. [CrossRef]

2. Chichra, P.N. New subclasses of the class of close-to-convex functions. Proc. Am. Math. Soc. 1977, 62, 37-43. [CrossRef]

3. Ali, R.M. On a subclass of starlike functions. Rocky Mt. J. Math. 1994, 24, 447-451. [CrossRef]

4. Dziok, J.; Srivastava, H.M. Certain subclasses of analytic functions associated with the generalized hypergeometric function. Integral Transforms Spec. Funct. 2003, 14, 7-18. [CrossRef]

5. Gao, C.-Y.; Zhou, S.-Q. Certain subclass of starlike functions. Appl. Math. Comput. 2007, 187, $176-182$. [CrossRef]

6. H. Silverman, A class of bounded starlike functions, Int. J. Math. Math. Sci. 1994, 17, 249-252. [CrossRef]

7. Singh, R.; Singh, S. Convolution properties of a class of starlike functions. Proc. Am. Math. Soc. 1989, 106, 145-152. [CrossRef]

8. Srivastava, H.M. Some Fox-Wright generalized hypergeometric functions and associated families of convolution operators. Appl. Anal. Discret. Math. 2007, 1, 56-71. 
9. Srivastava, H.M.; Frasin, B.A.; Pescar, V. Univalence of integral operators involving Mittag-Leffler functions. Appl. Math. Inf. Sci. 2017, 11, 635-641. [CrossRef]

10. Srivastava, H.M.; Yang, D.-G.; Xu, N.-E. Subordination for multivalent analytic functions associated with the Dziok-Srivastava operator. Integral Transforms Spec. Funct. 2009, 20, 581-606. [CrossRef]

11. Srivastava, H.M.; Prajapati, A.; Gochhayat, P. Third-order differential subordination and differential superordination results for analytic functions involving the Srivastava-Attiya operator. Appl. Math. Inf. Sci. 2018, 12, 469-481. [CrossRef]

(c) 2019 by the authors. Licensee MDPI, Basel, Switzerland. This article is an open access article distributed under the terms and conditions of the Creative Commons Attribution (CC BY) license (http:// creativecommons.org/licenses/by/4.0/). 